\title{
Criterios de medición de excelencia del recurso humano en la Atención Primaria de Salud. Caso: Chile
}

\author{
Carlos Sánchez \\ Universidad de Chile
}

\begin{abstract}
Resumen
El presente estudio busca evaluar los criterios que determinan la excelencia de un Centro de Salud Familiar utilizados por el Ministerio de Salud. Se utilizó una metodología cualitativa, para ello se realizaron dos focus groups segmentados en personas directivas y personas no directivas de la Atención Primaria de Salud. Luego de realizado los focus groups, se revisaron fuentes de información secundaria específicamente en relación a los criterios de evaluación del recurso humano. En los resultados obtenidos se identificaron ocho características que se asocian a un centro de salud familiar de excelencia y se proponen dos nuevos criterios para evaluar al recurso humano al momento de certificar a un centro modelo.
\end{abstract}

Palabras clave: Recurso humano, salud pública, Atención primaria.

\section{Measurement criteria Human Resource Excellence in Primary Health Care. Case: Chile}

\begin{abstract}
This study seeks to evaluate the criteria that determine the excellence of a Family Health Center used by the Health Ministry. A qualitative methodology was used and two focus groups were conducted with managers and non-managerial people belonging to the Primary Health Care. Then, secondary sources were reviewed specifically in relation to the evaluation criteria of human resources. The obtained results identify eight characteristics that are associated with a family health center of excellence and two new proposed criteria for evaluating the human resource to certify a model center.
\end{abstract}

Keywords: Human resources, public health, primary care.

\section{Criterios de medición de exce- lencia del recurso humano en la Atención Primaria de Salud.}

La Atención Primaria de Salud (APS) surge como estrategia en 1978 en la Conferencia Internacional de Atención Primaria de Salud, en donde los gobiernos, los profesionales sanitarios y los implicados en el desarrollo, generan la estrategia que busca proteger y promover la salud para todas las personas del mundo al año 2000, quedando establecidos en la Declaración de Alma-Ata (Organización Panamericana de la Salud, 1978). En la declaración, se considera a la APS como un integrante del sistema completo de asistencia sanitaria, que incorpora: la promoción de la salud, la prevención de daños a la

\footnotetext{
*Dirección de correspondencia [Correspondence address]:

Carlos Sánchez, Universidad de Chile

E-mail: csanchezt@med.uchile.cl
}

salud, la rehabilitación; centrado en privilegiar la participación de las personas de la comunidad en el cuidado de la salud. Se pone énfasis también al rol que juegan los equipos de salud a cargo de la comunidad, y como estos deben ser capaces de poner en práctica las políticas de Estado. Esta misma declaración establece en el VI punto, que la APS es la asistencia sanitaria esencial basada en métodos y tecnologías prácticos, científicamente fundados y socialmente aceptables, puesta al alcance de todos los individuos y familias de la comunidad mediante su plena participación y a un costo que la comunidad y el país puedan soportar, en todas y cada una de las etapas de su desarrollo con un espíritu de autorresponsabilidad y autodeterminación. La atención primaria forma parte integrante tanto del sistema nacional de salud, del que constituye la función central y el núcleo principal, como del desarrollo social y económico global de la comunidad. Representa el primer nivel de contacto de los individuos, la familia y la comunidad con el sistema nacional de salud, llevando lo más cerca posible la atención de salud al lugar donde residen y trabajan 
las personas, y constituye el primer elemento de un proceso permanente de asistencia sanitaria.

A comienzos de la década de mil novecientos noventa, los Gobiernos de la época abordan lo que va a ser el cambio del modelo de la APS en Chile, dando inicio entonces, al Modelo biopsicosocialSalud Familiar importado de países desarrollados. La mirada biopsicosocial viene a romper los paradigmas del antiguo modelo, ahora la relación médica es mucho más cercana; el médico y el equipo de salud participan junto con el paciente en la definición del problema, aporta sus propios elementos culturales en la manera en que interroga, habla y escucha al paciente. Siempre modifica el campo de observación, al darle un sentido a lo que el paciente trae, sea un síntoma o una molestia, y juntos organizan una determinada entidad o patología (Ceitlin y Gómez. T, 1997).

Siguiendo con la línea de cambio de modelo, el Ministerio de Salud de Chile estableció entre los ejes centrales para el avance de la estrategia, al recurso humano (Organización Panamericana de la Salud, 2000). Esto se fundamenta en que el principal instrumento de trabajo se centre en los conocimientos, habilidades y destrezas de este recurso. En este sentido se deberán considerar la elaboración de planes nacionales de acción de recursos humanos para la salud, con metas y objetivos específicos, un conjunto apropiado de indicadores y un sistema de seguimiento, con la finalidad principal de fortalecer las capacidades integradas de atención primaria de salud y de salud pública, así como de garantizar el acceso a poblaciones y comunidades sub-atendidas (Organización Panamericana de la Salud y Organización Mundial de la Salud, 2007).

Es cierto que contar con un equipo de personas de salud estable y capacitado es un beneficio para cada Centro de Atención Primaria, hoy existen desafios importantes que resolver para la población chilena: principalemente las causas de muerte son cardiovasculares y enfermedades degenerativas; los chilenos (especialememente las mujeres) tienen una esperanza de vida superior a los 80 años (Ministerio de Salud, 2013a), los principales problemas de salud están asociadados al tabaquismo, obesidad, sedentarismo y de mal nutrición (Encuesta Nacional de Salud, 2003-2010). En este escenario es necesario establecer nuevas formas de medición para los centros de salud familiar y para el personal de salud, más exigentes, que asegure poder dar respuesta a los desafios planteados, entregar una mejor atención, una mayor resolutividad y una óptima promoción de salud y de bienestar para toda la comunidad.

De esta forma, la presenta investigación tiene co- mo objetivo evaluar los criterios utilizados por el Ministerio de Salud, relacionados con el recurso humano, que determinan la excelencia de un Centro de Salud Familiar. Para cumplir este objetivo, se busca describir la dotación y capacitación del recurso humano utilizados por el Ministerio de Salud de Chile como criterios para seleccionar a los "Centros de Salud Familiar de Excelencia"; identificar los elementos que se relacionan con la excelencia de una Centro de Salud Familiar; formular nuevos criterios para evaluar el Recurso Humano que aseguren la excelencia de los Centros de Salud Familiar; y por último, establecer brechas entre los criterios de evaluación del Recurso Humano existentes y los nuevos criterios identificados.

\section{La concepción biopsicosocial de la sa- lud y los recursos humanos.}

La aplicación de una concepción biopsicosocial de la salud exige considerar a la persona integralmente e inserta en su medio ecológico: familia, barrio, trabajo, escuela, medio ambiente. También exige considerarla a través de todo su ciclo vital, lo que significa adecuar la oferta asistencial a las diferentes etapas de la vida.

El modelo está basado en los siguientes principios (Ministerio de Salud, 2013b:18-22):

a) Centrado en las personas

b) Integralidad

c) Continuidad del cuidado

El Modelo de Atención Integral con Enfoque Familiar y Comunitario se plantea orientado a proporcionar a los individuos, familia y comunidad, el cuidado de la salud de manera integral, continua, oportuna, eficaz, accesible y de calidad. En este contexto, la salud se entiende como un bien social y la red de salud como la acción articulada de la red de prestadores, la comunidad organizada y las organizaciones intersectoriales. Implementar el modelo desde esta perspectiva, implica el desafío de la transformación de los consultorios a centros de salud familiar.

La concepción del nuevo modelo en la atención primaria de salud, descansa no sólo en un rediseño organizacional, si no que requiere de equipos de salud capaces de asumir el cuidado de salud de población a su cargo de manera integral utilizando el enfoque familiar de salud y comunitario. Esto significa, rescatar la importancia del desarrollo de habilidades clínicas, sociales y de gestión local en la 
formación de los recursos humanos de salud (Ministerio de Salud, 1997a). El gran desafío del sistema de salud primario, entonces, se encuentra en el desarrollo de habilidades y destrezas que permitan a los equipos de salud analizar el proceso de saludenfermedad centrado en las personas y desde una perspectiva sistémica.

Estos esfuerzos deben ser guiados por diferentes principios orientadores, que dan un marco referencial para establecer al recurso humano como el elemento central de la mirada biopsicosocial, para esto, la Organización Mundial de la Salud entregó algunos ejes relevantes de acción: (1) Los recursos humanos son la base del sistema de salud: El desarrollo de los recursos humanos en salud conforma la base social y técnica de los sistemas de salud y de su mejoramiento. La contribución de los trabajadores de la salud es un factor esencial de la capacidad del sistema de salud de asegurar el acceso equitativo a servicios de calidad a toda la población. (2) Trabajar en salud es un servicio público y una responsabilidad social: El trabajo en salud es un servicio público y un bien social de gran importancia para el desarrollo humano. Es necesario un equilibrio entre los derechos y responsabilidades sociales de los trabajadores de salud y los derechos sociales y responsabilidades de los ciudadanos que merecen la atención y el derecho a la salud. (3) Los trabajadores de salud son protagonistas de su desarrollo: El desarrollo de los recursos humanos en salud es un proceso social (no exclusivamente técnico), orientado a mejorar la situación de salud de la población y la equidad social, a través de una fuerza de trabajo bien distribuida, saludable, capacitada y motivada. Este proceso social tiene como uno de sus protagonistas a los trabajadores de la salud (Organización Panamericana de la Salud, 2005).

Dentro de ese marco, uno de los retos claves en la Atención Primaria es el de dotar a los sistemas de salud con recursos humanos en número suficiente y adecuadamente capacitados, de manera que sea el lugar donde se puedan resolver la mayoría de los problemas sanitarios que padece la comunidad. En efecto, la renovación de la APS exige una transformación profunda en los programas de formación de los profesionales de la salud, así como la expansión y diseminación del conocimiento sobre este nivel de atención.

Al respecto, el avance de la estrategia de renovación comportará la necesidad de contar con más profesionales con formación específica, es decir, capacitados para desempeñar un conjunto de funciones específicas de la atención primaria. Este conjunto de conocimientos y habilidades se diferencia de los del resto del sistema y se deberá adecuar al nuevo contexto configurado conforme a la estrategia de la APS. Asimismo, el énfasis que dicha estrategia ejerce sobre la equidad de acceso y la calidad de los servicios requiere sistematizar los procedimientos y el trabajo en red, como poderosos instrumentos para aumentar la capacidad resolutiva de los equipos al fomentar la coparticipación y la corresponsabilidad de sus integrantes (Nebot et al., 2009).

Toda renovación sistémica en los servicios de salud conlleva profundas transformaciones en los modelos de gestión y de atención, generando forzosamente demanda de nuevas competencias y modificando en consecuencia la estructura de los recursos humanos. Uno de los elementos definitorios de la educación permanente, es su orientación hacia y desde el trabajo cotidiano, la programación del aprendizaje desde la realidad laboral y la orientación hacia la mejora del desempeño utilizando el enfoque de competencias profesionales. Sin duda, las competencias constituyen en la actualidad una conceptualización y un modo de operar en la gestión de recursos humanos que permiten mejorar la articulación entre gestión, trabajo y educación. Del análisis de sus diversas definiciones puede concluirse que las competencias son características permanentes - conocimientos, actitudes y habilidadesque la persona demuestra cuando realiza con éxito una actividad, sea laboral o de otra índole, que propicia a un "recurso humano adecuado".

\section{Situación del Recurso Humano en Chile.}

En Chile, como en el mundo, ha surgido una creciente preocupación en torno al recurso humano, el que es crucial para la producción de cuidados para la salud. Para esto se estableció un marco referencial (Ministerio de Salud, 1997b), que tiene por objetivo fortalecer y promover las políticas públicas en recursos humanos tanto para el sector privado, como para el público.

Los temas más relevantes en materia de recursos humanos relacionados a las Políticas de Salud en Chile, se podrían agrupar en diferentes categorías: reducción de costos e incremento de la eficiencia, mejoramiento y gestión del desempeño, planificación del personal (equidad en la distribución; educación y capacitación), condiciones de trabajo, desarrollo de capacidades de planificación y creación de políticas en recursos humanos para la salud (Martínez y Martineau, 1998; Dussault y Dubois, 2003). En cualquier caso, el ámbito de las políticas relacionadas con los recursos humanos para la salud es muy amplio e involucra la coordinación de distintos sectores.

La reforma de salud — cuyo objetivo principal es 
el mejoramiento de la salud de la población- considera un rediseño del sistema en todos sus componentes, incluyendo como uno de sus puntos el "desarrollo y fortalecimiento del recurso humano" (Sandoval Orellana, 2004), reconociéndose de esta forma el importante papel jugado por los trabajadores para "el impulso, puesta en marcha e implementación de una reforma de salud". De hecho, la ley de Autoridad Sanitaria incluye consideraciones respecto de las asignaciones de desarrollo, estímulo al desempeño colectivo, de acreditación individual, de estímulo a la función directiva, de turno, de responsabilidad, y a la promoción en la carrera funcionaria.

Sin embargo, el tema de los recursos humanos para la salud ha sido mencionado por algunos autores como uno de los desafíos pendientes de la reforma (Méndez, 2009; Román et al., 2006), ya que a pesar de haber presentado una oportunidad para los trabajadores de la salud de mejorar sus condiciones salariales y de trabajo, según Méndez (2009:276) se enfocó en la productividad y en los incentivos, postergando así, entre otros, la desigual distribución del recurso humano en salud, la necesidad de reducir la concentración de especialidades y prestaciones de alta complejidad, y el aumento de la migración de profesionales.

De esta forma, los autores señalan que la calidad del servicio, su eficacia, efectividad, accesibilidad y viabilidad dependen directamente del desempeño de quien lo entrega. Y a su vez, este desempeño está determinado por las políticas y prácticas que definen por ejemplo, el número de empleados, su calificación, su distribución (en términos territoriales, de nivel de atención, sector en el cual se desempeñan) y sus condiciones laborales. Al mismo tiempo, dichas políticas y prácticas deben considerar los compromisos políticos, las necesidades de financiamiento y sobre todo, las necesidades de la población. Según los autores, la no consideración de todos estos factores y la ausencia de una planificación estratégica, en este sentido, pueden llevar a desequilibrios de la fuerza de trabajo tales como el inestabilidad de la gestión de los recursos humanos y los objetivos nacionales de política, disparidades cualitativas (brechas entre programas de entrenamiento y los requerimientos de la política de la salud del país), distribución desigual de la fuerza de trabajo (entre áreas geográficas, categorías de profesiones, establecimientos de salud y especialidades). En cualquier caso, en la formulación de las políticas antes mencionadas se deben tener en consideración ciertas características de la actividad de los recursos humanos del sector, como la naturaleza intersectorial de muchos de los problemas relacionados con éstos, así como la variedad de participantes envueltos. Otra característica importante a considerar es el rezago entre la toma de decisiones y el resultado, siendo necesario un período de tiempo considerable para obtener cambios en la fuerza laboral de salud, por ejemplo por el número de años requeridos para formarla o el impacto en el largo plazo de tendencias poblacionales que pueden tener efectos en las necesidades futuras de la población (envejecimiento). Por otra parte, está la fuerte dominancia profesional y la interdependencia de distintas categorías de profesionales, además del importante rol del Estado como empleador principal entre otras consideraciones.

En cualquier caso, se debe reconocer que los temas de recursos humanos han estado presentes en la agenda política durante los últimos años en Chile, destacando en esta materia, los esfuerzos que se han realizado en contar con una dotación acorde a las necesidades del sistema sanitario. Según datos del Ministerio de Salud, ésta ha crecido en un $130 \%$ en 9 años. Si solo se observan los últimos cuatro años (de 2010 a 2013), dicha dotación se ha incrementado en un $35,5 \%$ en la APS. Este importante crecimiento ha estado acompañado de un mayor financiamiento en términos del monto per cápita. De acuerdo a datos de la Organización Panamericana de la Salud del año 2013, los países de la región habrían logrado una razón de densidad de recursos humanos de salud de 25 profesionales por cada 10.000 habitantes (Centro de Políticas Públicas UC, 2014).

La formación continua para profesionales especialistas en APS en nuestro país ha sido débil y no ha existido un plan de formación con incentivos sostenidos para impulsar este desarrollo. Este aspecto ha sido una de las dimensiones más frágiles de la Reforma de Salud. En este sentido, se han detectado dificultades en el ámbito de los recursos humanos: 1) una evidente falta de médicos para la APS; 2) los médicos existentes tienen insuficientes competencias para resolver en forma efectiva los problemas que enfrentan y muchas veces no están preparados para el trabajo con equipos multidisciplinarios; 3) bajo reconocimiento del trabajo en el nivel primario, lo que se expresa en los bajos sueldos; y 4) la insuficiente importancia asignada a la APS en la formación profesional y de especialidad (Centro de Políticas Públicas UC, 2014).

Dussault y Dubois (2003:17) señalan cuatro recomendaciones o 'recetas' para el desarrollo de políticas relacionadas al personal de salud: la primera indica que las políticas en recursos humanos para la salud deben ser comprehensivas, considerando todos los aspectos relacionados y por lo tanto yendo más allá de la mera administración de los recursos. Lo segundo, es que estas políticas deben reflejar la naturaleza integrada, interdependiente y sistemáti- 
ca de los diferentes componentes involucrados. En tercer lugar, que dado el rol crítico y estratégico que cumplen los recursos humanos en las organizaciones de salud, este tipo de políticas requieren de tomadores de decisiones y administradores proactivos. Finalmente, que la movilización de todas las partes interesadas es un elemento clave en el desarrollo, implementación y evaluación de estas políticas.

\section{Metodología}

La presente investigación tiene un carácter exploratorio y utiliza una metodología cualitativa. Se realizaron 2 grupos focales segmentadas en personas Directivas (Estamento profesional) y personas no Directivas (Estamento profesional y técnicosadministrativos) de la Atención Primaria de Salud. Las personas pertenecientes a estos grupos tienen entre 5 y 20 años de experiencias en APS.

Los participantes del grupo directivo fueron seleccionados bajos los criterios de reconocimiento y experiencia en atención primaria por un comité de expertos. Para contrastar y complementar los resultados obtenidos en este grupo, se realizó un segundo grupo focal, donde los participantes fueron escogidos por conveniencia, es decir, aquellos que tenían la disposición y la voluntad de participar en esta investigación. Ambos focus group fueron desarrollados en el mes de junio del año 2014.

Las profesiones de salud que se relacionan con la APS son: Medicina, Kinesiología, Fonoaudiología, Enfermería, Obstetricia y Puericultura, Nutrición y Dietética, Tecnología Médica, Psicólogos y Terapia Ocupacional. Como estamento no profesional, se encuentran administrativos y técnicos de nivel superior. En este estudio participararon: 2 Kinesiologos, 1 médico, 2 médicos de familias, 1 matrón y 1 Técnico de nivel superior (Grupo Focal no directivo).

A continuación, se revisaron fuentes de información secundaria: en este sentido, se exploró Información relevante para la investigación, específicamente en relación a los criterios de evaluación del recurso Humano.

Finalmente, para la estrategia de análisis, se codificó la información y se agrupó en grandes categorías para facilitar la presentación de datos y el análisis de esto.

En cuanto a los criterios para evaluar el recurso humano, se identifican las siguientes categorías.

\section{Resultados}

\section{Centro de Salud de Excelencia.}

El nuevo modelo de atención, de carácter integral, familiar y comunitario, está orientado a que la atención de salud sea un proceso continuo que se centra en el cuidado integral de las familias, preocupándose de la salud de las personas antes que aparezca la enfermedad, entregándoles herramientas para su autocuidado. Su énfasis está puesto en promover estilos de vida saludables, en fomentar la acción multisectorial y en fortalecer la responsabilidad familiar y comunitaria para mejorar las condiciones de salud. En caso de enfermar, se preocupa que las personas sean atendidas en las etapas tempranas de la enfermedad, de modo de controlar su avance y evitar su progresión a fases de mayor daño y de tratamientos más complejos.

En este sentido, existe un consenso entre los participantes del focus group en que un eje trascendental de todo Centro de Salud Familiar (CESAFAM) es el enfoque sanitario. Lo importante del enfoque sanitario radica en una mirada integradora que se desarrolle desde el modelo de atención, y que el enfoque sea trabajado desde la política pública como una estrategia y no como una tarea aislada de los procesos; tal como lo señala un participante

Para poder pensar el dispositivo sanitario que respondan a este modelo óptimo o de excelencia [...] [Hay que pensar que] todos los elementos técnicos van por un carril [...] y el tema financiero va por otro lado, que tiene que ver con el fomento de la individualidad, de la toma de decisiones particulares, de la libertad de elección [...] Y por tanto, eso es disonante con este discurso técnico y programático.

Un aspecto que surgió de los participantes, fue que se debía acotar lo sanitario solo a la atención, en este sentido se apunta a incorporar todos los componentes que sean necesarios para entregar una atención de salud de calidad, y es en este punto en donde surge uno de los desafíos que tiene la APS: la Resolutividad.

Yo creo que es importante en un centro de excelencia o de calidad es la resolutividad [...] Todos dicen faltan especialistas en la atención primaria. Faltan ambulancias, faltan hospitales, faltan cosas biomédicas. Todos. Y el problema de la resolución es de la APS [...] El problema es de la APS y la verdad que la resolución es del sistema sanitario. 
Cuadro 1: Categorías Focus Groups: Centros de Salud de Excelencia

\begin{tabular}{ll}
\hline Categorías & Subcategorías \\
\hline \hline Sanitario & Cobertura de salud sobre la población. \\
& Resolutividad en la APS. \\
& Especialidad de Medicina Familiar. \\
\hline Comunidad & Participación de los agentes comunitarios en las decisiones. \\
& Trabajo con redes sociales. \\
& Trabajo intersectorial. \\
\hline Recursos Humanos & Equipos de salud con compromiso. \\
& Equipos de salud transdisciplinario. \\
& Capacitación. \\
\hline Infraestructura y tecnología & Modelo arquitectónico moderno. \\
& Lugar para el equipamiento de salud. \\
\hline Sectorización & Población a cargo suficientemente identificada. \\
\hline Financiamiento & Relacionadas a las Políticas Remuneracionales. \\
& Modelo de financiamiento definido de acuerdo a las necesi- \\
& dades reales. \\
\hline Liderazgo & Gestión y Dirección del centro. \\
& Presencia del gestor en la comunidad y en la red. \\
\hline \hline & Contar con un Equipo Directivo. \\
\hline \hline
\end{tabular}

Fuente: Elaboración propia

Cuadro 2: Categorías Focus Groups: Centros de Salud de Excelencia

\begin{tabular}{ll}
\hline Categorías & Subcategorías \\
\hline \hline Clima Organizacional & Plan de calidad de vida para el recurso humano. \\
& Trabajo en equipo. \\
\hline Perfiles y competencias & Directivos, Encargados de Programa y Equipo de salud en \\
& general. \\
& Promover la igualdad de Género. \\
& Competencias de ingresos. \\
\hline Capacitación & Temas relacionados con el Modelo de Salud Familiar / Me- \\
& dicina Familiar. \\
\hline Dotación & Definición de dotación acorde al plan de salud comunal. \\
\hline \hline & Concursos Públicos. \\
& Fuente: Elaboración propia
\end{tabular}

Principalmente lo que quiere establecer el participante es que para fortalecer lo sanitario se debe mejorar la resolutividad y esto incorpora a todo el equipo de salud, que hoy en día desde una mirada integral, al médico de familia. Y es aquí donde los participantes que tienen dentro de su formación a la especialidad médica mencionada, concuerdan en que la medicina familiar es un aporte en lo resolutivo. Sin embargo, llevándolo a la práctica propiamente tal de la APS, el problema radica en la falta de dar soluciones o respuestas a los problemas de salud que tiene la comunidad.

Hubo un acuerdo general en que los CESFAM deberían estar insertos en una red asistencial, que contenga un trabajo colaborativo en la implementación del modelo en cada centro, tal como lo menciona un profesional de la salud
No puede ser desintegrado de un trabajo colaborativo, transdiciplinario, sanitario, educacional, de promoción de salud. Y me parece que la segmentación sanitaria pura genera más divisiones que suma o sinergias entre salud y recurso. Por lo tanto este centro de excelencia no lo veo como un ente aislado sino que lo veo dentro de una organización mucho más compleja, intra salud e intra otras organizaciones de la comunidad y de la administración y del desarrollo social y comunitario.

Uno de los ejes centrales que constituye una base sustentable para la APS, es la participación de la comunidad en la situación de salud de sus territorios y localidades. En este sentido, existe consenso entre ambos focus group en que es necesario que exista una mayor participación y empoderamiento 
de la comunidad en las decisiones estratégicas de sus centros de salud. Para esto se proponen diferentes espacios de acción:

a) Que cada directorio o equipo directivo de los CESFAM al menos debe estar constituido por algún agente comunitario.

b) Darle mayor empoderamiento a los consejos consultivos ${ }^{1}$.

Lo que se busca con activar a la comunidad a participar de la APS es poner sus problemas locales de salud como ejes de acción prioritarios y fortalecer el control social de la acción de los CESFAM. Es por ello, que no es casual que al momento de consultar por un CESFAM de excelencia, el eje comunitario se encuentre como base del modelo. Una de las participantes señala lo siguiente:

Yo lo asocio a escaleras y procesos. Y creo que cada peldaño de la escalera me hace mucho sentido lo que dice en relación a la participación. Creo que efectivamente uno de los escalones de arriba tiene que ver con cuando uno efectivamente tiene la comunidad empoderada participando, gestionando, congestionando y haciendo control social también.

Con esto, además, se pretende ir capacitando a la comunidad en el modelo, ir incorporando y fortaleciendo el liderazgo de los agentes comunitarios, esto queda claro con la siguiente cita: "entregar a la comunidad un poco de responsabilidad, de herramientas para que ellos generen sus formas también de tomar parte de la prevención, de la promoción de la salud".

Por otro lado, los equipos hacen una autocrítica dado que reconocen que no se generan los espacios necesarios de trabajo con la comunidad. Es por ello que, de forma transversal en ambos grupos, se insta a los equipos de salud a generar una participación activa con la comunidad. A estos elementos, se suma, el trabajo de integración que se tiene con la red de salud e intersectorial que tiene como gran tarea mejorar las condiciones sociales en las cuales las personas viven y trabajan. El trabajo intersectorial deberá proveer las herramientas necesarias para el cuidado de la salud de una población determinada, enmarcada por la política pública sanitaria. Esto queda expuesto en la siguiente cita:

\footnotetext{
${ }^{1}$ El Consejo Consultivo de Usuarios tiene la función de asesorar al Director del establecimiento en la fijación de las políticas y en la definición y evaluación de los planes institucionales.
}

Creo que un centro de excelencia tiene que estar abocado a eso, a tener metas sanitarias locales, territoriales y para poder trabajar metas territoriales que vayan concatenadas con las nacionales, porque no podemos dejar la mirada nacional, tiene que ver un trabajo intersectorial y comunitario fuerte y eso hoy día no se está valorando. Estamos pegados todavía en el box.

En lo que respecta a las personas, es importante establecer algunos elementos para la excelencia de un CESFAM. En este contexto, surge de los focus group un componente de éxito: compromiso de los equipos de salud. Con ello se obtienen importantes avances del modelo de salud familiar, obteniendo impacto en el trato al usuario, resolutividad, cercanía, clima organizacional, entre otros elementos.

Una situación particular es cuando se trata el compromiso de los médicos y especialmente de los médicos de familia en la atención primaria, y aquí el grupo de profesionales y técnicos lo hace notar aún más. La discusión se centra principalmente en la línea ética de lo público y privado y de los problemas que genera el mercado de la salud cuando los incentivos económicos privados superan a los público, obteniendo como problema una baja tasa de permanencia de los médicos en la APS y la importante brecha entre oferta y demanda de médicos generales y especialistas en Chile.

Sumado a lo anterior, ambos grupos refuerzan la estrategia de contar con equipos multidisciplinarios capaces de fortalecer la APS en Chile.

También se rescata la capacitación como base del modelo y como uno de los principales componentes que pueden influir en el personal de salud positivamente. Una directiva lo explica y lo detalla en la siguiente cita:

Debiera haber un equipo con competencias para desarrollar el modelo, con competencias para trabajar en la Atención Primaria. Y el modelo, en marco de sus principios y de la capacitación comunitaria (...) debiera tener planes de capacitación continua, de verdad. Porque hoy día, si bien es cierto, hay capacitación porque hay un mandato, pero no siempre son de verdad. Son para cumplir las 40 horas.

Con respecto a la infraestructura y la tecnología, es importante mencionar que durante en el discurso presidencial del año 2014 se da un fuerte impulso a la Atención Primaria, comprometiendo así, la construcción de 132 SAPUS de Alta Resolutividad, 100 nuevos CESFAM, y 100 nuevos Centros Comunitarios de Salud (CECOF) a lo largo del país. Este 
aporte en infraestructura se suma a los 566 Centros de Salud Familiar, 1.168 Postas de salud rural, 228 Servicios de atención primario de urgencia, 112 Servicios de urgencia rural y a los 166 Centros comunitarios de salud familiar existente hoy en la Red de Asistencial chilena (Molina, 2014).

De los participantes, existe un consenso en cuanto al rol y al aporte de contar con un lugar físico acorde, moderno, con tecnología y cercano. Claramente queda expresado en la siguiente frase realizada por una participante: "Debemos partir por tener una buena infraestructura, una infraestructura moderna, un modelo arquitectónico, de acuerdo a lo que significa trabajar el modelo de salud integral".

Por otra parte, la incorporación de tecnologías a la APS tiene como propósito mejorar la calidad de la atención; importante han sido los avances en los casos de las tecnologías de información y comunicación para la salud, un ejemplo claro de esto es la implementación del registro clínico electrónico en la APS.

Los aportes en la telemedicina también han sido un elemento favorable para el modelo, y a pasar que es un procedimiento que no se encuentra en todos los lugares, los resultados han sido importantes. Junto a lo anterior, los médicos de la especialidad de medicina familiar que participaron en la investigación, proponen seguir fortaleciendo los avances en la telemedicina, lo que es significativo para la resolución diagnóstica de los pacientes, lo que va en directo beneficio de la comunidad.

La estrategia de sectorización tiene como principal objetivo el conocimiento de familias y comunidad en general de los equipos de salud. Se espera que mientras menor sea el número de familias, la posibilidad de conocerlas en profundidad sea mayor; esto con el objetivo de hacer realidad el enfoque biopsicosocial. Este equipo es el responsable de otorgarle los cuidados primarios de salud y de conectarlo con el sistema sanitario en su totalidad.

El cambio de la mirada biomédica a la biopsicosocial, implica un acercamiento a la comunidad con criterios de transferencia de poder, en el cual el paciente pasa a ser un usuario proactivo, con manejo de información, y con un equipo que desarrolla habilidades de comunicación acordes al contexto actual (Subsecretaría de Redes Asistenciales, 2008).

Este elemento generó gran discusión en el grupo directivo, en cambio en el grupo de profesionales y técnicos, fue un elemento más de análisis que se trató brevemente y no generó mayor profundización o debate. De esto, se rescata que en el grupo formado por los directivos, el tema de la sectorización se analizó con mayor profundidad, concluyendo que ha generado efectos negativos no deseados para el modelo. Pensando en un centro óptimo, se establece que en lo que respecta a este ámbito, existen aún tareas por resolver y se incentiva a retomar el rumbo para la que fue diseñada la estrategia. Un directivo lo señala de la siguiente forma:

Me genera algún grado de ruido que por ejemplo, en la lógica de la salud familiar se haya centrado fuertemente en la sectorización y en una parte importante de los centros lo que visualizó, y dejándome caer a diferentes horas, es ver a veces tres centros al interior de uno, que es un efecto no deseado, en realidad, de generar la idea de tener más pequeños para poder tener población a cargo y que ojalá lo conozca. O sea, un centro de salud familiar óptimo tiene que tener su población a cargo suficientemente identificada.

En esta misma línea, se deja en claro que la sectorización es parte del modelo y es por ello que otra participante señala al respecto que:

\begin{abstract}
Respecto a la estructura del modelo, a la sectorización que es división del trabajo, porque no es más que eso, a mí me parece que durante muchos años le pusimos mucho énfasis, como que la sectorización fuera el modelo, y la sectorización no es el modelo, es una forma de trabajar.
\end{abstract}

Por último, se espera que la tarea de fortalecer la sectorización debe venir desde las políticas públicas emanadas del Ministerio de Salud. Desde esta orientación una directiva realizó una crítica al rol que estaba cumpliendo este organismo:

\section{El Ministerio puso un compromiso de gestión que tenía que ver con sectorización, entonces se con- fundió, y lo digo porque muchos lo expresaron así cuando yo he visitado centros particularmente en la sexta región. Entonces tenemos que sectorizar porque el modelo dice que hay que sectorizar.}

El sistema de financiamiento para la APS consiste en un modelo de asignación de los recursos en salud a través de un monto por persona, el cual se ajusta según el riesgo, aislamiento geográfico, pobreza de las personas atendidas. Dicha asignación, que es particular para cada comuna, se estructura con un componente basal y otro variable ${ }^{2}$

\footnotetext{
${ }^{2} \mathrm{El}$ componente variable consiste en una modificación porcentual de acuerdo a factores como ruralidad, pobreza y acceso a la atención de salud; y la asignación de monto fijo considera edad (mayores de 65 años) y desempeño difícil.
} 
En este sentido la investigación arroja que en ambos grupos existe disconformidad del monto per cápita que se utiliza actualmente, indicando que dicho modelo de asignación no cubre totalmente los costos reales que presenta el sistema, tal como lo señala un participante:

Porque de verdad el per cápita actual no funciona y creo que podría ser el doble que uno recibe en este minuto. Si se lleva a cifras OCDE la verdad es que el desafío es enorme, no es posible financiar una atención de excelencia con 6 dólares por persona. Lo mínimo son 18 dólares por persona. Cualquier país OCDE funciona con eso.

Los participantes mencionan que habitualmente se encuentran en situaciones muy complejas, por ejemplo, la población solicita ambulancias, nuevos equipamiento y los funcionarios requieren capacitación y nuevas políticas remuneracionales, entonces los directivos se enfrentan a decisiones importantes para la salud pública y para el avance del modelo.

En esta línea resalta la necesidad de contar con una Política de Remuneración acorde a valores de mercado, ya que existen profesiones que tienen una remuneración más baja en comparación al sistema hospitalario público y privado, consultas privadas, etc. Esta política debe tener incentivos que motiven a la permanencia del personal sanitario. Se espera que exista un mayor aporte presupuestario desde el nivel central, en esta materia.

Dentro de cualquier proceso o implementación de las políticas públicas, se hace necesaria la existencia de personas que cuenten con las capacidades, habilidades y liderazgo, que sea capaz de llevar el proceso de implementación del modelo de salud, a través de direcciones o corporaciones de salud y de todos los centros de salud a lo largo de Chile. Estas personas, conforman un equipo directivo, encargado de llevar el rumbo de cada centro de salud, tomar las decisiones, negociar los recursos con sus direcciones de salud, entre otras innumerables tareas en relación a la certificación y a la calidad de estas instituciones.

Según establecen los participantes, el éxito o no, de un buen centro de salud, depende del liderazgo que ejerzan sus directivos, así lo señala una académica: "Necesitamos directivos competentes que puedan conducir efectivamente el modelo (...) Sí hay un directivo de salud que ocupa ese car-

Junto con lo anterior, existe otro mecanismo de financiamiento que se aplica a 52 comunas cuyo tamaño poblacional es inferior a 3.500 habitantes. Estas comunas reciben una cantidad de recursos predeterminada, independiente del número de usuarios actuales que se atiendan en el sistema (Centro de Políticas Públicas UC, 2014). go por un tema político partidista, me parece la perversión misma de un sistema sanitario del país".

Con lo anterior, se busca que los directivos asuman su rol con liderazgo y acción, ya que, las decisiones que tomen van, a afectar la salud de su territorio. Se espera además, que el líder sanitario tenga presencia en el territorio y tenga contacto y una relación directa con la comunidad.

Es importante recalcar la experiencia de los directivos que participaron en la investigación, ya que aportan estrategias de acción que pueden servir a otros centros del país. Estos aportes son en la línea de obtener un equipo directivo que sea un aporte a cada centro, es así que una directiva entrega su estrategia de éxito la cual es contar con a lo menos con 3 personas competentes y con habilidades para conformar un equipo directivo. La participante lo establece en la siguiente cita

Estoy convencida que un centro de salud familiar tiene que tener al menos tres directivos [...] Necesito una directora o director del centro de salud, necesito un profesional que sea jefe de SOME y que me administre el $80 \%$ de los recursos porque mi agenda, que es responsabilidad del equipo directivo y necesito además una subdirección y en mi definición, la subdirección es aquella que trabaja gestionando los recursos de adentro del centro.

Lo que se espera entonces del equipo directivo, es que se debe contar con personas con competencias para desarrollar el modelo y para trabajar en la Atención Primaria, como lo señala un participante profesional: "Yo creo que ahí está la base de todo, si hay buenos líderes, buenos jefes de sector, buenos directivos, esos equipos van adelante, pero cuando no, cuesta más".

Como último elemento que contribuye a un centro de excelencia, se identifica a las investigaciones que se realizan en los centros con apoyo de instituciones universitarias nacionales e internacionales, fundaciones y otras instituciones que aportan al desarrollo científico en materia de salud poblacional. En este sentido, la ejecución de investigaciones locales se ve favorecida por el apoyo de instituciones académicas, que ayudan a la búsqueda de recursos adicionales, y el aporte de recursos docentes calificados en investigación, de manera de asegurar la calidad de los estudios que se realicen, y que estos produzcan impacto positivo en el mejoramiento de los procesos locales, así lo señala el documento "En el Camino a Centro de Salud Familiar". La investigación operativa y la investigación acción son metodologías adecuadas para la generación de co- 
nocimiento local, ayudando tanto a equipos como a las comunidades en la adquisición de información útil para la toma de decisiones y generación de propuestas locales, que puedan además, retroalimentar políticas públicas.

Principalmente en este ítem se rescata el apoyo de las universidades, así lo señala un directivo:

Para mí un centro de excelencia tiene que hacer investigación operativa (...) Las universidades, los centros formadores debieran invertir en que los equipos de atención primaria aprendan a hacer investigación de buena calidad. Tenemos mucho insumo y mucha información, y creo que eso también debiera ser un espacio privilegiado.

Lo que se espera de la investigación en los CESFAM, es que ésta debe ser capaz de traducir la experiencia en excelencia, en atención primaria y salud familiar, en resultados concretos, reproducibles y publicables que se puedan replicar en otros lugares.

\section{Criterios para evaluar el recurso hu- mano en un Centro de Salud Familiar.}

El clima organizacional se refiere al ambiente de trabajo propio de la organización, dicho ambiente ejerce influencia directa en la conducta y el comportamiento de sus miembros. En tal sentido, se puede afirmar que el clima organizacional es el reflejo de la cultura más profunda de la organización, determinando la forma en que el trabajador percibe su trabajo, su rendimiento, su productividad y satisfacción en la labor que desempeña (Acevedo, 2008).

En relación a este punto, los grupos son claros en señalar que como primer elemento de análisis, al momento de hablar de recursos humanos, surge el clima organizacional y la calidad laboral en la que día a día funcionarios de la APS se desempeñan; en consonancia con lo anterior, un profesional señala lo siguiente: "Es fundamental que las personas que trabajan adentro tengan una satisfacción, que tengan un clima adecuado, que tengan oportunidades de desarrollo, porque de lo contrario ningún modelo puede avanzar cuando el clima laboral no es el adecuado".

En este sentido, se destaca que es un avance ir reconociendo las potencialidades de los integrantes de cada centro, ir fortaleciendo el trabajo en equipo, y esa tarea es de un directivo que sea capaz de liderar el modelo al interior de este. Así lo señala una integrante del focus group: "Es importante eso del reconocimiento del equipo como tal, como una máquina, un engranaje".

Es importante mencionar que en el segundo grupo focal, existió un debate en relación al trabajo en equipo y el rol que cumplen los médicos de familia como un integrante de los equipos de salud, existieron dos miradas al respecto. Es inherente que una visión corresponde a los médicos de la especialidad versus la mirada de los otros profesionales. En este contexto, una integrante establece que los médicos de familia no se integran a los equipos, $\mathrm{y}$ que además, no cumplen con las expectativas con las que esperan a momento de hacer devolución de su beca, ella lo señala en la siguiente cita

La experiencia de médicos que han llegado después de hacer su beca en salud familiar. Y nosotros esperamos todos al médico de salud familiar que venga a saludarnos y veo que la postura no es mucho la que cambia, porque todavía sigue en su escritorio, todavía no quiere salir a ver a pacientes afuera. Nosotros: doctor, por favor, atiéndanos a este paciente. Bueno, qué hacemos con esta familia. Tienes que esperar.

Desde la otra perspectiva, y siempre enmarcado en el trabajo en equipo, un participante médico de familia señala que esta profesión es la que tiene mayor carga laboral, lo que genera poca integración en los equipos, dado el escaso tiempo que tienen para participar en reuniones de sector, u otras instancias de trabajo integrativo. El participante menciona lo siguiente:

Son los profesionales que atienden a la mayor cantidad de gente, están sobre explotados por la organización, son los que no participan en las reuniones de sector, son los que no los consideran en ninguna otra cosa. Entonces es como un apego, desapego. Están, pero por favor no estén. Es una cosa muy curiosa porque los mismos equipos los rechazan y ellos rechazan a los equipos, pero los equipos los necesitan y ellos necesitan a los equipos.

Finalmente, se establece ir perfeccionando todas aquellas acciones que vayan en mejora de la calidad de vida. Es rescatable que ambos grupos fortalezcan la integración de los equipos pensando en un marco organizacional de excelencia.

El siguiente punto trata sobre los perfiles y competencias que tienen los profesionales que trabajan e ingresan a la atención primaria de salud. Como contexto general, existe una disconformidad del 
proceso de formación de pregrado de los profesionales, ya que, pareciera ser que los perfiles de egreso no entregan formación para la APS, lo que va generando problemas para la organización, tales como: desmotivación de los funcionarios, problemas de atención al usuario, alta rotación de profesionales, entre otros. Un participante rescata la situación:

Desde la formación de pregrado no hay competencias para trabajar en la atención primaria. No hay competencia para trabajo en equipo, no hay competencia para trabajo solidario, no hay competencia (...) Me parece que hay una insuficiencia competencia de los profesionales que están trabajando en la Atención Primaria, no por voluntad de ellos, sino porque sus procesos de formación son inadecuados.

Es por ello que los participantes concuerdan que es necesario definir un perfil mínimo de competencias de ingreso a los centros de salud, perfiles que deben concursarse públicamente. Se promueve los concursos públicos en los cargos directivos y jefaturas intermedias, acorde a los perfiles establecidos por cada municipio, con esto además se promueve la igualdad de condiciones y de género para los cargos que sean de esta índole. Lo anterior permitirá tener un modelo organizacional sólido y validado por la comunidad.

Por último, es importante mencionar, que una participante directiva invita a realizar la reflexión sobre la humanización del trato y el talento humano, que cada uno de las personas tiene al interior de cada organización; es rescatable esta apreciación, ya que motiva a la reflexión sobre el rol que cada persona cumple en el modelo de salud familiar y comunitaria:

Entonces yo creo que ahí lo que nos falta también es transitar hacia una conceptualización de los recursos humanos para la atención primaria que dan cuenta de su rol, de su misión, de su función recolocando un concepto que es el del talento humano... Más que los recursos humanos como los estamos mirando de manera habitual y cotidiana... Y olvidamos que los recursos humanos es un concepto más fino, más delicado, más reflexivo, que tiene que ver con qué expertise, qué destrezas, (...) Desde ahí uno es realmente es el servidor público con todo ese tecnicismo que aprendió en algún lugar.

Al analizar la capacitación funcionaria, se debe mencionar que ésta queda mandatada por la Ley $\mathrm{N}^{\circ}$
19.378, que establece el Estatuto de Atención Primaria de Salud Municipal, sumado a eso el perfeccionamiento es uno de los elementos que se utiliza como medio o indicador al momento de calificar a un consultorio como CESFAM, y luego a un CESFAM de excelencia.

Principalmente lo que se mide en este punto, es que la dotación establecida en el plan de salud local de cada municipio se capacite según lo establece la normativa. Sin embargo, los participantes señalan que la actual normativa requiere recomendaciones o actualizaciones, así lo destaca una directiva: "yo creo que el estatuto de atención primaria requiere un par de recomendaciones nuevas, una de ellas en el ámbito de capacitación. Yo creo que nos estamos quedando cortos con los cinco días de capacitación".

En este sentido, la necesidad de capacitar en el modelo de salud familiar y en medicina familiar (en caso de profesionales médicos) apunta a entregar, mejorar y/o establecer formación al personal de salud que permitan crear equipos multidisciplinarios capaces de dar respuesta a las problemáticas del sistema sanitario chileno. Un profesional del área señala lo siguiente:

Entonces debiera haber un equipo con competencias para desarrollar el modelo, con competencias para trabajar en la Atención Primaria. Y el modelo, en marco de sus principios y de la capacitación comunitaria y debiera ser además de la competencia, debieran haber planes de capacitación continua, de verdad. Porque hoy día, si bien es cierto, hay capacitación porque hay una mandata, pero no siempre son de verdad. Son para cumplir las 40 horas.

Se espera entonces, que la capacitación sea una herramienta que se encuentre en cada proceso de la implementación del modelo. Con esto se fortalece el rol que tienen las personas dentro de cada centro de salud y se promueve la formación continua, la actualización en salud pública y en estrategias que favorezcan la promoción de la salud y la calidad de vida de la comunidad. Para cerrar, mencionaremos lo que establece una participante en cuanto a la formación: 
Falta formar a ese equipo a escala humana y por ahí el tema del trato, y todo lo que viene. Y por ahí tanta problemática interna también. Porque yo creo que ahí falta mucho meter capacitación o gestión, no sé, a escala humana, para que la gente de verdad haga un cambio de switch y teniendo ese centro que sería maravilloso, la gente que está dentro de verdad diga: chuta, yo tengo que ser maravilloso en mi manera de ser y de trabajar aquí para poder estar.

$\mathrm{Al}$ igual que en el punto anterior, los aspectos normativos a la dotación se encuentran establecido en la Ley 19.378. Este elemento es uno de los dos requisitos (otro es capacitación) para postular al programa de CESFAM de Excelencia, que se implementó durante la gestión 2010-2014 en Chile.

Ahora bien, dentro de los resultados obtenidos de los grupos focales, se puede destacar que ambos grupos llegan a la conclusión que en la APS la dotación de profesionales de las carreras de la salud como de otras áreas de las ciencias sociales, administrativas, es insuficiente. En este sentido, señalan, además, que se debe fortalecer una política pública de recursos humanos que involucre nuevos incentivos para permanecer en el sistema público, aquí un directivo señala lo siguiente:

\begin{abstract}
Algo que no hemos tocado y que es fundamental es el tema que tiene que venir una política de recursos humanos como país [...] Y yo creo que eso está en este minuto en lo central del problema en recurso humano del sector público. O sea, políticas públicas que tienen que ver con la formación, que tienen que ver con el tema de educación. Qué universidades estamos recibiendo, qué profesionales estamos formando o a quien recibimos.
\end{abstract}

Es importante analizar que los grupos concuerdan que los equipos de salud están rindiendo al máximo de su fuerza laboral, es ahí la importancia de no sobrepasar la barrera de la sobreexplotación del recurso humano. Es por ello que se espera que a nivel Ministerial existan recursos presupuestarios y estrategias a nivel de políticas públicas para ir dotando a los centros de no tan solo profesionales médicos, sino además abrir los espacios para que otras profesiones participen y se hagan parte del Modelo de Salud Familiar y Comunitario, así lo indica una directiva:
Los equipos no se atreven a definir una dotación, porque dicen "no, no me la van a aprobar" y no tienen fundamentos para que se la aprueben. Entonces el equipo también... vuelvo a las competencias de liderazgo y de cómo conducir, en para reformular las dotaciones, no con lo que dijo el Ministerio, sino que una dotación de acuerdo a las necesidades locales de territorio.

El llamado entonces es que el nivel central asuma la responsabilidad de estas nuevas necesidades, invirtiendo en formación y políticas de incentivo, y que estas a su vez, involucren a todos los funcionarios del centro, para que asuman la responsabilidad pública. Con lo anterior se espera, por ejemplo, reducir la brecha existente a nivel de profesionales médicos y poder contar con una dotación adecuada en términos de números y de calidad. En esta línea, una participante señala:

Si antes habían dos médicos, ahora deben haber por lo menos seis, para poder hacer efectivamente medicina familiar. Lo mismo pasa con el resto de los funcionarios, tienen que aumentarse en un porcentaje importante, cercano al 30, $40 \%$ de equipo de salud, no sólo en números, sino que en tipo de profesionales, pero también en números.

Otro punto destacado de los grupos, corresponde a la dotación de profesionales médicos con especialidad en Medicina Familiar. En este sentido, se espera que exista una estrategia y una definición política sobre la importancia y lo trascendental que es contar con médicos especialistas en los centros de salud primaria, ya que según estos mismos señalan, es la formación adecuada para responder oportunamente al modelo biopsicosocial.

Finalmente, otro elemento de análisis que se mencionó, era que toda dotación debía cumplir con criterios de selección previamente establecidos en un perfil y que éstos debían postularse por medio de concurso público. En este sentido se hace necesario regularizar las nuevas contrataciones en los municipios de Chile.

\section{Conclusiones y recomendacio- nes.}

En la actualidad, el solo hecho de contar con una dotación y la verificación de ésta, y cumplir con las horas de capacitación mandatadas por ley, como criterios de evaluación del recurso humano, no aseguran por sí sola la excelencia de un centro de 
salud que utilice el enfoque biopsicosocial y comunitario, ya que carece de otros elementos esenciales relacionados con el eje central de un Centro de salud Modelo.

De acuerdo al análisis, es posible describir que existen 2 criterios con los que el Ministerio de Salud evalúa al recurso humano en la APS: la dotación y la capacitación. En materia de dotación, ésta se entiende como aquella definida por la comuna en cuestión versus la constatación en terreno del personal existente en cada uno de los estamentos del establecimiento. Junto con ello, se evalúa la capacitación y formación continua de los equipos de salud, mediante el programa de capacitación anual, desarrollado con un $80 \%$ de participación del personal, verificado con lista de asistencia firmada por los participantes.

Los elementos identificados para un centro de salud de excelencia son:

- Enfoque sanitario.

- Participación comunitaria.

- Los recursos humanos.

- Infraestructura y tecnología.

- La sectorización y reconocimiento de la población.

- Un sistema de financiamiento acorde a las necesidades.

- Líderes sanitarios y comunitarios.

- Centros que realicen investigación con apoyo de instituciones universitarias y otros organismos.

Estos ocho elementos entregan valor a un centro de salud familiar que busca mantener a una población sana y vinculada con el medio en donde se desenvuelve. A partir del análisis de los ocho elementos, es posible construir un modelo que clarifique la labor y el valor central que debe tener un centro de salud familiar de excelencia. Para ello, se trabajará con el modelo CANVAS, diseñada por Osterwalder $^{3}$; herramienta conceptual que, mediante un conjunto de elementos y sus relaciones, permite expresar la lógica mediante la cual una organización genera valor. Osterwalder definió una ontología consistente en una estructura de nueve blo-

\footnotetext{
${ }^{3}$ Osterwalder cuenta con Maestría en Ciencias Políticas en la Universidad de Lausana, donde en 2004 había obtenido su doctorado en Sistemas de Información de Gestión en virtud de Yves Pigneur con la tesis titulada "The Business modelo Ontología. Mayor información en http://alexosterwalder.com/
}

ques temáticos, que agrupan las principales variables de un negocio. El modelo destaca como una importante herramienta en la comunicación, el análisis y la evaluación de modelos de negocio, así como en la innovación. Los ejercicios que resulten de aplicar esta herramienta pueden conducir a verdaderas innovaciones en una forma sencilla y clara para quienes participan en el proceso creativo y para quienes deben implementarlo (García, 2010). CANVAS, permite, analizar tanto los modelos privados o empresariales, como además, el sector público.

En todo ciclo de una política pública, una de las etapas que refleja el éxito o fracaso de la misma, es vista en la etapa de la evaluación, en este sentido lo que se busca con acciones como las de certificación de los centros de salud a CESFAM o el programa de CESFAM de excelencia realizado durante los últimos años, es verificar el desarrollo del modelo de salud familiar y comunitario en los centros de salud a lo largo del país, desde una mirada de política pública. Son escasos los estudios que existen al respecto; en esta materia se rescata la investigación de evaluación del proyecto de Salud Familiar ANCORA UC, estos centros son gestionados gracias a la alianza de la Pontificia Universidad Católica de Chile, el Servicio de Salud Metropolitano Sur Oriente y el Ministerio de Salud, a diferencia de los gestionados por la red pública municipal o los pertenecientes a los servicios de salud.

Es en esta materia, donde la contribución de la presente investigación viene a entregar una propuesta a la certificación existente de los centros de salud al momento de evaluar, como criterio al recurso humano. Esta contribución es teniendo como precedente que existen centros con un mayor o menor desarrollo del modelo a lo largo de todo el país, por ello se deja un espacio de acción que se deberá determinar a nivel local.

Esta propuesta, viene a complementar las recomendaciones que se han desarrollado a lo largo de estas conclusiones, siendo un eje clave que da sentido al presente estudio de caso y que puede ser un elemento transcendental a la hora de certificar los centros de salud a lo largo del país.

La propuesta cuenta con cuatro criterios de evaluación acompañados de sus verificadores:

Esta propuesta, entrega un marco referencial para las políticas públicas en salud, diseñando las estrategias y las acciones pertinentes para avanzar hacia la mejora continua y permanente de la calidad de los centros de salud familiar, en concordancia con las estrategias de la "cobertura universal" en salud que estableció la OPS/OMS durante el año 2014: ampliar el acceso equitativo a servicios de 
Figura 1: Modelo Canvas de un Centro de Salud de Modelo

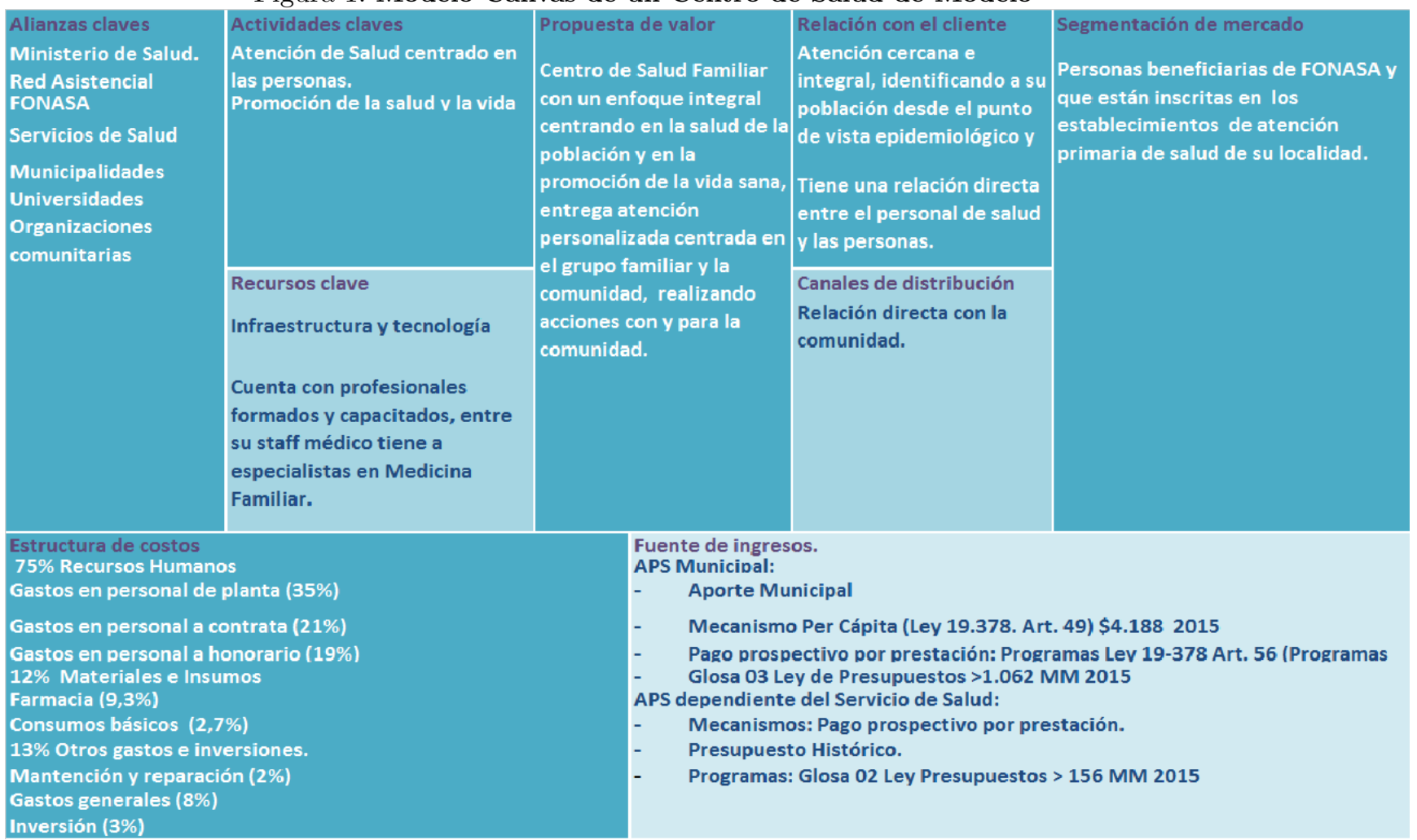

Fuente: Elaboración Propia

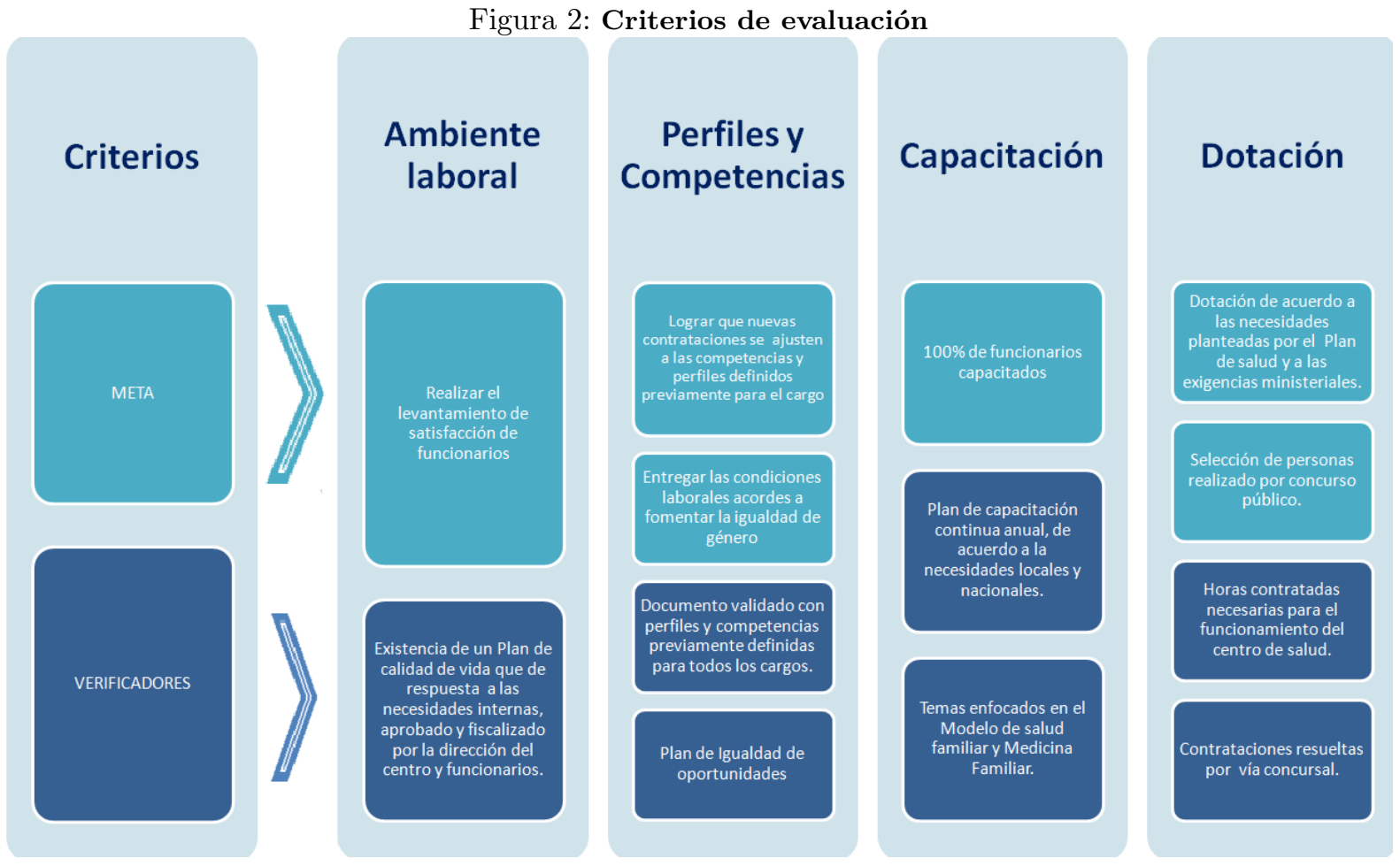

Fuente: Elaboración Propia 
salud integrales, de calidad, centrados en las personas y las comunidades; fortalecer la gobernanza; mejorar el sistema de financiamiento y fortaleciendo la intersectorialidad para abordar determinantes sociales de salud. Todos componentes que entrega esta investigación, que distinguen a un centro de salud familiar y a su recurso humano, centrado en el modelo de salud familiar y comunitaria.

La propuesta complementa las recomendaciones del estudio, en donde se quiere destacar por ejemplo el de potenciar una Política de formación integral de especialistas de Medicina Familiar para la APS, acorde a los desafíos del país, focalizada las demandas de salud que tiene la población de acuerdo a las realidades locales. Esta es una estrategia país que viene a disminuir la brecha existente de profesionales médicos en el sistema primario.

Junto con ello, otra recomendación es la de fomentar la participación comunitaria, orientada a tener una intervención relevante en el Plan de Salud Comunal, presupuestos, consejos y comités locales, y a generar un trabajo conjunto con la red intersectorial (escuelas, juntas de vecinos, comunidades y asociaciones indígenas, entre otras organizaciones). Esta participación debe estar enmarcada en la actual política pública (Ley $\mathrm{N}^{\circ} 20.500$ ) sobre Asociación y Participación Ciudadana en la Gestión Pública en donde se establece la institucionalización de la participación ciudadana en la gestión pública y además determina el marco referencial de la asociatividad en Chile.

Por otra parte, como tercer eje de acción, se recomienda actualizar el per cápita a un valor acorde a la canasta de prestaciones actual, considerando la situación epidemiológica y demográfica, con ello se pretende reducir la brecha de aportes a la APS.

Junto con ello, se propone incentivar los convenios con universidades nacionales y extranjeras, que favorezcan la investigación en la atención primaria. Esto permitirá establecer una política de alianza del Estado a través de sus Universidades, con los centros de la APS, obteniendo trabajos investigativos con los diferentes datos con los que cuenta cada dentro de salud. Esta acción, entregará información relevante para la toma de decisiones y para la elaboración o desarrollo de políticas públicas orientadas mejorar la salud de la población.

Finalmente, se destaca que al momento de analizar brechas entre los criterios existentes y los propuestos, es importante rescatar que la propuesta que entrega el estudio, tiene por objetivo resguardad el bienestar y el desarrollo de las personas que trabajan en la atención primaria, ya que estos son un pilar fundamental para el desarrollo de un cen- tro de salud modelo con enfoque integral. La brecha que queda por trabajar, es la incorporación de acciones y estrategias que permitan un desarrollo integral de las personas pertenecientes a la APS, potenciando planes de desarrollo institucional, incentivando y motivando la permanencia de funcionarios en la institución y colaborando con el bienestar y felicidad de estos.

\section{Referencias}

Acevedo, J. (2008). Clima Organizaconal. Santiago.

Ceitlin, J. y Gómez. T (1997). Medicina de Familia: la clave de un nuevo modelo. IM\&C, Madrid.

Centro de Políticas Públicas UC (2014). Fortalecimiento de la atención primaria de salud: propuestas para mejorar el sistema sanitario chileno. Santiago.

Dussault, G. y Dubois, C.-A. (2003). Human resources for health policies: a critical component in health policies. Human resources for health, $1(1): 1$.

García, J. F. (2010). Innovación en modelos de negocio: La metodología de Osterwalder en la práctica. Revsita MBA EAFIT, pp. 30-47.

Martínez, J. y Martineau, T. (1998). Rethinking human resources: an agenda for the millennium. Health Policy and Planning, 13(4):345-358.

Méndez, C. A. (2009). Los recursos humanos de salud en Chile: el desafío pendiente de la reforma. Revista Panamericana de Salud Pública, 26(3):276-280.

Ministerio de Salud (1997a). De Consultorio a Centro de Salud. Santiago.

Ministerio de Salud (1997b). Diseño e Implementación de las Prioridades de Salud. Santiago.

Ministerio de Salud (2013a). Indicadores Básicos de Salud. Santiago.

Ministerio de Salud (2013b). Orientaciones para la implementación del Modelo de Atención Integral de Salud Familiar y Comunitaria. Santiago.

Molina, D. (2014). Situación actual y desafíos de la atención primaria.

Nebot, C., Rosales, C., y Borrell, R. (2009). Desarrollo de competencias en atención primaria de salud. Revista Panamericana Salud Pública, pp. 176-183.

Organización Panamericana de la Salud (1978). Declaración de Alma-Ata. 
Organización Panamericana de la Salud (2000).
Funciones esenciales de salud pública. Madrid.

Organización Panamericana de la Salud (2005). Llamado a la Acción de Toronto: 2006-2015 Hacia una década de Recursos Humanos en Salud para las Américas. Toronto.

Organización Panamericana de la Salud y Organización Mundial de la Salud (2007). Documento de Posición de la Organización Panamericana de la Salud/Organización Mundial de la Salud. Washington, D.C.
Román, O., Acuña, M., y Señoret, M. (2006). Disponibilidad de médicos en Chile al año 2004. Revista médica de Chile, 134(8):1057-1064.

Sandoval Orellana, H. (2004). Mejor salud para los chilenos: fundamentos sanitarios, políticos y financieros de la necesidad y oportunidad de hacer una reforma al sistema de salud chileno. Cuad. méd.-soc.(Santiago de Chile), 43(1):5-20.

Subsecretaría de Redes Asistenciales (2008). En el Camino a Centro de Salud Familiar. Santiago. 